\title{
Lenguaje religioso y juventud(es) ¿cómo hablar del Dios de Jesucristo en el contexto educativo actual?*
}

\author{
Juan Pablo Espinosa Arce**
}

Recibido: 9 de febrero de 2016 • Aprobado: 23 de marzo de 2016

\section{Resumen}

La búsqueda de un lenguaje religioso que dialogue con la posmodernidad constituye una tarea irrenunciable para la Iglesia en vistas a su misión evangelizadora. Uno de los principales campos de acción es el mundo de la educación a partir de la Educación Religiosa Escolar (ERE). El presente artículo aborda la problemática de cómo hablar del Dios revelado en Jesucristo a la juventud y más específicamente a las juventudes, ya que éstas representan un grupo humano homogéneo el cual responde a diferentes códigos, simbólicas o interpretaciones de la vida. La propuesta busca por ende proponer algunas categorías o estructuras de discurso por medio de las cuales y como educadores de la fe podamos provocar una evangelización más encarnada, más consciente de los cambios sociopolíticos, religiosos y culturales a los que las juventudes se ven enfrentadas de manera de propiciar una renovada síntesis entre fe, vida y cultura.

Palabras Clave: lenguaje religioso, juventudes, educación religiosa escolar, teología, evangelización

* Artículo de Reflexión. Ponencia leída en el I Congreso Nacional sobre el Fenómeno Religioso en el Mundo Contemporáneo. Santiago de Chile 14-16 de Octubre, 2015.

** Licenciado en Educación y Profesor de Religión y Filosofía (Universidad Católica del Maule). Magíster en Teología Fundamental (Pontificia Universidad Católica de Chile). Docente de Ética Profesional (Centro de Formación Técnica - Instituto Profesional Santo Tomás, Sede Rancagua - Chile). Correo electrónico: jpespinosa@uc.cl 


\title{
Religious language and youth. How to speak of the God of Jesus Christ in the current educational context?
}

\begin{abstract}
The search for a religious language that dialogues with postmodernism is an indispensable task for the Church in view of its evangelizing mission. One of the main fields of action is the world of education from School Religious Education (ERE, in Spanish). This article addresses the issue of how to speak of God revealed in Jesus Christ to youth and more specifically to youths, as they represent a homogeneous group which responds to different codes, symbolic or interpretations of life. The proposal seeks therefore to suggest some categories or structures of discourse through which, and as educators of faith, we can cause a more incarnate, more conscious evangelization of the social, political, religious and cultural changes to which the youths are faced in order to stimulate a renewed synthesis between faith, life and culture.
\end{abstract}

Keywords: Religious language, youth, school religious education, theology, evangelization.

\section{Langage religieux et jeunesse (es): comment parler du Dieu de Jésus-christ dans le contexte éducatif actuel ?}

\section{Résumé}

La recherche d'un langage religieux qui dialogue avec la postmodernité constitue une tâche à laquelle ne peut renoncer l'Église en vue de sa mission évangélisatrice. L'un des principaux champs d'action est le monde de l'éducation à partir de l'Education Religieuse Scolaire (ERS). Cet article aborde la problématique de comment parler du Dieu révélé en Jésus-Christ à la jeunesse et plus spécifiquement aux jeunesses, puisque celles-ci représentent un groupe humain homogène qui répond à différents codes, symboliques ou interprétations de la vie. La proposition cherche donc à proposer quelques catégories ou structures de discours au moyen desquelles, et comme éducateurs de la foi, nous puissions provoquer une évangélisation plus incarnée, plus consciente des changements sociauxpolitiques, religieux et culturels auxquels les jeunesses doivent faire face pour favoriser une synthèse renouvelée entre foi, vie et culture..

Mots-clés: Langage religieux, jeunesses, éducation religieuse scolaire, théologie, évangélisation. 


\section{Introducción}

Hoy el tema del lenguaje constituye uno de los grandes campos de estudio en la filosofía, la teología y el desarrollo de las ciencias de interpretación o hermenéuticas. Es más, su estudio "es asunto central de la cultura del siglo XX (...) la nuestra [época] es en lo cultural una era hermenéutica, que se interroga sobre el sentido de las palabras y de los textos" (Morales, 2004: p. 107). Esta pregunta por el sentido, sobre el qué quiere expresar tal o cual disciplina del saber, en este caso la teología, y cómo lo hace la Iglesia en la evangelización, constituye a nuestro juicio un desafío latente. Esto porque sabemos que la llamada época de la cristiandad se caracterizó por el predominio de la Iglesia en Europa y que llevaba como centro el que las realidades terrenas carecían de autonomía frente a una Iglesia con injerencia total en lo político, lo económico y lo social, éste época terminó por desvanecesre.

Gracias al Concilio Vaticano II acontece la conciencia de que la Iglesia debe abandonar el paradigma de cristiandad lo cual la lleva a buscar nuevas formas para la evangelización de una sociedad cada vez menos creyente. Y es en esta nueva evangelización que se hace necesario el uso de un nuevo lenguaje religioso que presente el mensaje del Evangelio y de la praxis eclesial de manera cautivante y que permita el proyecto de la recomposición del creer. La experiencia de Dios debe ser explicitada de tal manera que provoque la síntesis entre fe, vida y cultura, de tal manera que nuestros interlocutores puedan percibir la presencia de un Dios que no es indiferente a nuestra historia. Y uno de esos interlocutores más críticos son los jóvenes. Es más, a nuestro juicio ya no podemos hablar de la juventud, sino que debemos hablar de juventudes, grupos humamos que responden a diferentes códigos, simbólicas o interpretaciones de la vida. Es más y a juicio de Duarte (2000), dicho concepto permite "construir miradas más integradoras y potenciadoras de lo juvenil" (p. 60).

La realidad cada vez más compleja y abierta a los cambios que experimenta la sociedad postcontemporánea, la presencia de un nuevo contexto social en donde "la realidad se ha vuelto para el ser humano cada vez más opaca y compleja" (Aparecida, 36), también tiene como consecuencia repensar la fe de los jóvenes ante este nuevo escenario sociocultural y religioso. En un estudio organizado primero por la Pontificia Universidad Católica de Chile y luego replicado 
por la Universidad Católica del Maule (Talca-Chile) llamado “Jóvenes, cultura y religión"1 (2006 - 2013), se nos muestran interesantes elementos para diagnosticar o tratar de comprender la realidad creyente actual de tal manera de provocar la reflexión para repensar algunos elementos pedagógico-teológicos para poder hablar del Dios de Jesús, tarea perenne de la Iglesia, en este nuevo contexto. Vamos a enumerar algunos de ellos.

1. En relación a los principios que orientan la vida del joven, es interesante comprobar que el respeto a los demás, la dignidad de las personas y la búsqueda de la felicidad y de la equidad social son elementos relevantes para la consecución de los proyectos de vida de los nuevos actores sociales. Es un dato interesante porque se vendría a derribar el mito o el prejuicio de que las juventudes actuales viven de la apatía y del individualismo. Quizás los movimientos estudiantiles que se generan desde el llamado Movimiento pingüino (2006) hasta las grandes manifestaciones del 2011 - 2012 son signo de que existe una "resistencia de los jóvenes a ser simplemente olvidados" (Silva, 2006: p.69). El paradigma del sujeto olvidado, de la marginación y exclusión social que se hace tanto de los jóvenes como de los ancianos, es una de las características propias de esta civilización mundial basada en la praxis de exclusión como lo denunció el Papa Francisco en el encuentro con los jóvenes argentinos con ocasión de la Jornada Mundial de la Juventud en Río de Janeiro el año 2013.

2. En relación a la pregunta de cuál es el sentido que los jóvenes le atribuyen a $\mathrm{su}$ fe, es interesante notar que un alto porcentaje sostiene que la vive porque se siente apoyado por Dios. ¿Refleja esta respuesta un sentimiento de vacío? ¿Existe en los jóvenes, en nuestros estudiantes, niños y jóvenes una necesidad clara de sentirse apoyados y validados tanto como personas a la vez que como miembros de una comunidad humana? ¿Cuál es la realidad del centro educativo hoy? Los niños y jóvenes pasan un alto número de horas en los establecimientos educacionales, quedándoles poco tiempo para el compartir gratuito con sus familias, y al revés, los tutores llegan a las casa a altas horas del día y el encuentro familiar se ha diluido lentamente. ¿Cómo la religión y

1 En el caso del estudio realizado en la PUC es relevante la síntesis y la interpretación teológica que realiza Joaquín Silva en su artículo “Jóvenes y religión: cuando la crisis es motivo de esperanza" (2006). En el caso del estudio realizado en la UC del Maule ver los tres informes realizados entre los años 2007, 2010-2011 y 2012-2013. Las características de las poblaciones de encuestados en estos estudios en los que se encuestó a cerca de 1400 jóvenes entre ambas Casas de Estudios las hemos extraído tanto del artículo de Silva como de los informes de la UCM. 
la creencia en un ser superior permite que el joven pueda completar aquello que le falta en el sentido del apoyo?

3. Un tercer elemento y que es central para la comprensión de la espiritualidad y la religiosas actual en el mundo de las juventudes es el lugar que ocupa la institución Iglesia en su relación con Dios. La imagen de un importante número de jóvenes hoy es que las instituciones religiosas y sus líderes son inconsecuentes. Se ha incrustado en el imaginario socioreligioso actual la consigna: Dios si, la Iglesia no. Es interesante notar que aunque la mayoría de la población del país se dice católica en virtud del bautismo, el tema de la identidad y de la pertenencia eclesial no está asumido o se ve afectado por las situaciones conocidas de escándalos al interior de la Iglesia vista mayoritariamente como institución. Entonces ¿cómo hablar de Dios o como vivir la fe si la relación con la comunidad creyente está debilitada? Si el axioma la fe supone comunidad creyente no es tomado en consideración ¿cómo la educación religiosa viene a completar aquello que falta? ¿Cómo está presente el tema de la representatividad de los líderes religiosos en los jóvenes? Silva (2006) sostiene que "las explicaciones de la distancia hacia la institucionalidad eclesial no hay que buscarlas sólo al interior de la Iglesia (...) probablemente los procesos de secularización, individualización, privatización, están siendo mucho más decisivos (...) los jóvenes han tomado distancia de todo tipo de instituciones" (pp. 87-86). Pero esto genera un problema, porque anteriormente sostuvimos que para muchos el sentido de la vida está en el encuentro con los demás y que la lucha conjunta por un ideal, por ejemplo la educación de calidad y gratuita, ha movilizado a muchos jóvenes a unirse en Centros de Alumnos $\mathrm{u}$ otras formas de representatividad. ¿Cómo compatibilizar entonces la apatía hacia la institución y los movimientos sociales? ¿Existirá alguna relación entre fe y política válida para las juventudes?

4. A propósito de lo anterior, de la posible relación fe y política, fe y movimiento social, incidencia del Evangelio en la demanda social, es interesante comprender que para los jóvenes no es difícil asumir que la palabra de Jesús de Nazaret representa la lucha en favor de la dignidad de los excluidos. Hay ansias por buscar caminos de libertad y de una Iglesia que sea la institución de la libertad y de la justicia así como la llamó J. B. Metz al formular su Teología Política. Pareciera ser que un renovado diálogo entre fe y justicia puede ser una de las vías interesantes para formular un nuevo lenguaje religioso por medio del cual se pueda hablar del Dios de Jesucristo a las juventudes. 
Pueden ser muchos más elementos con los cuales diagnosticar la situación de la creencia o de la increencia actual. Solo quedémonos con estos, que a nuestro entender son los más transversales o notorios. A partir de esto pasaremos a pensar los conceptos de lenguaje religioso, de juventudes y a proponer cinco elementos o categorías para pensar una nueva evangelización en el ámbito de la educación. Ellos son una teología y un lenguaje simbólico, narrativo, dialogante, del juego y del encuentro. Finalmente propondremos una síntesis de manera que sirva de pre-texto para continuar con la reflexión sobre un tema de relevancia tanto para educadores y en definitiva para la Iglesia.

\section{Para clarificar conceptos: lenguaje religioso y juventudes}

\subsection{Lenguaje religioso}

En relación al lenguaje en general y del religioso en particular, debemos partir de una convicción básica, esto es que es imposible no comunicar algo (axioma de la comunicación). Así tenemos un lenguaje verbal, no verbal, proxémico, analógico y también uno de carácter religioso. Ahora bien, para comprender a qué hacemos referencia cuando decimos lenguaje religioso, debemos sostener que "al hablar del lenguaje teológico y de la comunicación de la fe estamos hablando de la verdad del quehacer teológico" (Arteaga, 1997: p. 255), por ende para definirlo debemos hacerlo en el contexto de las tareas propias de la teología entendida como lenguaje sobre Dios y explicitación de la fe en categorías que pueden ser asimiladas por la experiencia humana.

En teología hablamos de analogías, es decir de la manera en la que se "nos permita hablar de Dios y de las cosas divinas de modo que nuestro lenguaje tenga sentido y no sea meramente ficticio o vacío. Analogía es una manera de emplear las palabras para que, en determinadas condiciones, digan algo acerca de lo que Dios es y hace" (Morales, 2004: p.115). La analogía la hemos de comprender en el escenario amplio de la Revelación, es decir, de la autocomunicación de Dios al hombre el cual se manifiesta condescendientemente es decir de una forma en la que su creatura puede entenderlo y responderle mediante su fe $(\mathrm{Cf}$. DV, 5). 
Junto con el tema de la analogía debemos considerar también el tema de las tareas de la teología que vienen a fundamentar también el sentido del lenguaje religioso. Dichas tareas son las de carácter positivo que es la escucha de la Revelación (en comunidad, en oración, lectura de la Palabra de Dios, etc), la función especulativa por la cual y una vez escuchada la Revelación explicitamos dicha fe en conceptos comprensibles que manifiesten el Misterio de Dios y finalmente el carácter práctico de la teología; es decir, su concreción en la pastoral, la catequesis, la evangelización, la liturgia, etc.

Al seguir con el tema de las tareas propias de la teología y de la Iglesia al momento de realizar su misión de hablar de Dios a los nuevos interlocutores, es necesario recordar que "la tarea más importante de la nueva evangelización consiste en volver a hablar de Dios, en hacer de nuevo a Dios, por así decir, materia de conversación" (Kasper, 2003: p. 284). El punto es que esto no es una tarea fácil porque la palabra "Dios" ha sido una de las más mal usadas a lo largo de la historia (M. Buber), con lo cual decir su nombre trae a la vez que elementos positivos, muchas consecuencias negativas (religión acomodaticia, clericalismo, terrorismo religioso, falta a la consideración de las fuentes mismas de una determinada religión, etc).

Pronunciar a Dios, hacerlo parte de la conversación educativa, pensar las demandas en este campo a la luz del Evangelio, constituyen tareas que deben realizarse y si ya se hicieron, volver a retomarlas. Este hacer de Dios parte de nuestras conversaciones, representa para Kasper (2003) una "nueva prioridad pastoral y cambio de paradigma" (p. 293), cambios que deben apuntar al anuncio, elemento central en la evangelización y en el lenguaje implicado en ella. Y uno de esos escenarios en los que se anuncia el Evangelio es la clase de religión y la catequesis, tanto parroquial como colegial. Y el mismo Kasper (2003) tiene una palabra esperanzada a propósito de esto cuando nos recuerda que "la clase de religión, a la que en su día se le asignó la tarea de iniciar en la fe, sigue teniendo importancia. Es una oportunidad que no debe desaprovecharse a la ligera" (p. 295), pero también es consciente de que "bajo las actuales condiciones escolares, solo en casos excepcionales puede llevar a cabo la iniciación en el ser cristiano y la fe de la Iglesia" (Kasper, 2003: p. 295). Lo último sostenido por Walter Kasper nos toca directamente como país, ya que en muchos colegios y liceos la clase de Religión ha sido reemplazada por temas de formación axiológica, ética o similares. Esto constituye otro desafío no menor pero que demandará un nuevo trabajo o reflexión. 


\subsection{Juventud y juventudes}

Una vez realizado este breve acercamiento al tema del lenguaje religioso, acerquémonos al concepto de juventud y juventudes. A juicio de Figueroa y Villena (2012) "el término juventud constituye algo complejo de definir, pues acercarnos al concepto implica considerar diversos elementos y variables" (p. 17). Juventud es un concepto equívoco, social o culturalmente hablando, esto porque representa una diversidad con lo cual se debe hablar de juventudes. Según las mismas autoras, cuatro características podrían definir las características de las juventudes vistas desde el mundo adulto. En primer lugar es una etapa transitoria entre la niñez y la adultez, o el logro de una mayor sensatez. En segundo lugar, es una etapa que más que producir riqueza para la sociedad absorbe recursos. Luego la visión de que los jóvenes son por un lado descontrolados y peligrosos y por otro lado que son sujetos incapaces, hasta representar un estorbo. Finalmente que son una población heterogénea. (Figueroa \& Villena, 2012)

Es interesante comprender que la juventud y el concepto de juventudes expresa una condición y una identidad social en la que se juegan las individualidades de los sujetos que componen estos grupos humanos. Con el término juventud y juventudes expresan un capital simbólico en el que se articulan relaciones en distintos ámbitos de interacción, por ejemplo la familia, la comunidad creyente, el mundo educativo o el mundo sociopolítico en los cuales se desarrolla la actividad del joven.

Las juventudes están también definidas por la interacción de una variada gama de condiciones, entre las que destacan el sexo (determinación biológica), el género (interacción psíquica en las relaciones recíprocas), los procesos de socialización tanto de pertenencia como de identidad en los que confluyen la aceptación o no en un determinado grupo humano, la experiencia cultural y la dimensión étnica, la creencia y los gustos personales, dan forma a un grupo heterogéneo al que no se puede abordad desde una sola mirada, sino que es necesario rescatar las múltiples voces e integrarlas a la conversación social, política, religiosa, educativa y cultural o como sostiene María Eugenia Villa (2011), las juventudes representan una "condición social diversificada que implica asumirla en plural" (p. 150).

Con este primer acercamiento, pasemos ahora a proponer algunas categorías por medio de las cuales se pretende repensar y asumir un nuevo lenguaje religioso de carácter plural y que dé más espacio a la sensibilidad y elementos extraídos de lo cotidiano. Estas categorías tienen la peculiaridad de ser "formas de discursos" como ha sostenido Ricoeur (1994), es decir, como formas originarias de la fe 
religiosa y la vehiculación de la misma en sus respectivos lenguajes. Asumimos esta categoría de la hermenéutica de Ricoeur (1994) porque vienen a "subrayar la función que juegan esas modalidades lingüísticas en la producción de los discursos (...) las formas de discursos son más que instrumentos de clasificación, son instrumentos de producción" (p. 59), es decir, al proponer estas categorías queremos volver a las fuentes mismas de la experiencia cristiana original de tal manera de favorecer lo propio de nuestro lenguaje religioso.

\section{Algunas categorías para repensar nuestro lenguaje religioso y teológico.}

\subsection{Teología y lenguaje simbólico}

La estructura simbólica es algo inherente a la fe cristiana. El Dios en el que creemos se ha hecho signo (sacramento), estructura visible y significativa en Jesús de Nazaret. La imagen y el símbolo nos ayudan pues a comprender el misterio de Dios, constituyéndose en una mediación necesaria. La estructura simbólica permite el "encuentro del hombre con la divinidad. Ese encuentro es mediado y celebrado en el mundo" (Boff, 1980: p. 106) en clave creativa y recreativa de estructuras y prácticas pastorales.

En la comprensión simbólica de nuestra fe, nos encontramos con clases de signos, entre los que encontramos la palabra hablada, la palabra escrita, las imágenes visuales de las cosas, y los sentimientos, gestos corporales, afectos. El itinerario formativo cristiano con ausencia de estructuras simbólicas "es de ordinario menos captado, menos aprehendido. Su poder emocional es, por consiguiente, considerablemente menor" (Nicolau, 1969: p. 26). Por lo tanto debemos promover una cultura teológica y pastoral más simbólica, más creativa y más imaginaria. En nuestra formación cristiana el símbolo asume un valor social, el cual

es el fundamento de las expresiones religiosas de una comunidad de creyentes. El símbolo, por su índole accesible a todos y universal, por su capacidad sugerente y por sus virtualidades de expresión individual y social, es algo que facilita el fenómeno sociológico de la religión. (Nicolau, 1969: p. 27). 
Desde esta socialización nacida de la experiencia simbólica se puede configurar un pensamiento teológico más concreto y encarnado en la realidad que posibilite que el joven y la comunidad creyente en general puedan experimentar el encuentro con el Dios de Jesucristo.

Un aspecto relacionado íntimamente con la experiencia simbólica de la fe lo constituye el concepto de imaginario teológico. El modo imaginario "es la modificación de lo real, la construcción de una distinta realidad, cuando la que vivimos no corresponde a nuestros deseos o aspiraciones" (Maccio, 1983: p. 21). Nosotros consideramos desde la experiencia teológica juvenil que el gran deseo o aspiración lo constituye la necesidad de un nuevo lenguaje teológico, más simbólico, más creativo y que favorezca la imaginación de lo real, la creación de nuevas categorías místicas, pastorales y teológicas que dialoguen de manera cercana con la realidad cotidiana del joven, de tal manera de provocar un mayor acercamiento entre el joven y su Iglesia y entre el joven y Cristo joven. El lenguaje que necesitamos postular como formadores de jóvenes, favorece a que se otorgue

mayor espacio a la sensibilidad y a la verdad. No aprisiona la manifestación de Dios. No es un lenguaje unívoco (como ocurre con el lenguaje conceptual) sino más bien polisémico. Esta riqueza de significados no da pie a la confusión; por el contrario, es una apertura a la manifestación del Misterio que tiene diversas significaciones. (Irarrázaval, 2000, pp. 190-191)

\subsection{Teología y lenguaje narrativo}

La narración constituye un elemento central en el lenguaje religioso y en la explicitación teológica. Gracias a ella y por medio de un lenguaje coloquial y testimonial, "se cuenta lo sucedido para descubrir ahí la acción salvadora de Dios. Se coloca al centro el relato, como formato específico para exponer las verdades acerca de Dios. Su inspiración y modelo es la narrativa bíblica. La teología narrativa quiere mantenerse en un lenguaje experiencial y vivencial más que argumentativo y abstracto" (Barriga, 1998, p. 9). Es interesante la mención del aspecto vivencial y experiencial, propio de la teología que quiere superar a la metafísica como una ciencia que no impacta en el hombre y proponer una que sea socialmente eficaz y que impacte en la vida cotidiana de la comunidad de creyentes, de la Iglesia que es forum de la memoria. 
Junto con esto, López Amozurrutia (2006) presenta la narración como un modelo o un método teológico, en donde aparece el arte de narrar, de expresar la experiencia de la fe la cual debe por una parte informar y por otra conmover. La narración para López Amozurrutia (2006) se comprende como "portadora de sentido" y como recuperación de la narración como "forma teológica originaria" de manera de ver al cristianismo como "comunidad de narración".

La narración se constituye como un poder en el sentido de conmover e informar, o como sostiene L. Duch (1979) un "poder evocador y transmutador" (p. 72 ), evocador porque se hace memoria de las acciones en favor de Dios para con su pueblo y cómo ese pueblo se constituye en el sujeto del diálogo salvífico. Esa memoria a su vez debe ser narrada, y en la ella "relatan los pequeñuelos y los oprimidos; pero éstos no sólo narran historias que les llevan constantemente a exaltar su propia opresión o minoría de edad, sino también historias peligrosas que buscan la libertad" (Metz, 1973: p. 229) . Gracias a la narración se constituye un sujeto capaz de hacer memoria contada acerca de la libertad de la que fue beneficiado. Es más, la narración posee una dimensión comunitaria en cuanto expresión de la solidaridad que se opone subversivamente al egoísmo de la sociedad tecnificada.

\subsection{Teología y lenguaje como diálogo}

El segundo aspecto es el diálogo. Al momento de ejecutar el diálogo se generan relaciones interpersonales entre dos interlocutores. Podemos ver nuestros rostros, escuchar nuestras voces, entrar en comunión con otro que me interpela. En esto, "proyectar el anuncio en términos de diálogo nos recuerda que el Evangelio -es decir, la misma persona de Jesucristo- no está determinado tanto por la geografía como por las personas de diferentes culturas, estratos sociales y preferencialmente desde el mundo de los excluidos y empobrecidos de nuestra sociedad. El anuncio se centra en la construcción de relaciones [vínculos] interpersonales que sean signos visibles del Reino del amor" (Liberti, 2007: p. 41). Así como las primeras comunidades cristianas se reunían en torno a la mesa de la palabra compartida y del pan partido para recrear y rememorar aquello que constituía su origen y fundamento, Jesucristo. Así las comunidades cristianas y aquellos que conduzcan sus etapas formativas, deben favorecer el encuentro cercano e igualitario entre todos sus miembros. 


\subsection{La teología como juego y encuentro}

Hemos realizado variados acercamientos a lo que debe ser lo sustancial en el proyecto formativo teológico y pastoral de las comunidades juveniles, a saber, el lenguaje teológico, las estructuras simbólicas e imaginarias, la narración y el diálogo. Ahora queremos adentrarnos en la comprensión de la teología vista como juego y como encuentro, ambos espacios de distención y de implicación socializada. Ver la teología como juego significa verla como un rito vivencial en el que cada uno de los implicados tiene su lugar y su rol dentro de su desarrollo. Por un lado, aquél que guía el diálogo y la formación y por otro el que es mi interlocutor, y que a su vez me constituye. Esta visión lúdica de la teología no debe caer en un conductivismo que vea al sujeto que viene a mi encuentro como una tábula rasa que no trae ningún conocimiento, sino que debemos valorar el alto potencial de experiencias personales y comunitarias que nuestros jóvenes han hecho de Dios. Este encuentro debe ser lúdico y dinámico, con leyes propias, pero que por estar presentes no coarten la libertad ontológicamente fundamental de los sujetos.

El juego asume una función ritual, esto porque "está lleno de funciones: crea propiamente el tiempo, articula y ordena la sociedad, integra los enigmas y vicisitudes de la precaria existencia y la sociedad, desde el cambio de ciclo, de estaciones, de poder, de situación o rol social y otorga una orientación a los días y las horas humanas" (Mardones, 2003: p. 168). El juego y el rito, así como el símbolo tienen la doble función, a saber, emotividad y socialización, ambas notas constitutivas de la esencia de nuestra fe y de la comunicación que debemos hacer de la misma. En esto, debemos sostener al segur a Huizinga citado en Mardones que “el juego está en la raíz de la cultura misma. El hombre jugaba, como el animal, antes de preguntarse, afirmar o negar la belleza, la verdad, la bondad, o al mismo Dios. Al principio está el juego, es decir, la actividad orientada por el impulso vital, sin finalidad utilitaria ni objetivo concreto" (Mardones, 2003, p. 169)

Ahora bien ¿cuál es la relación que se establece entre juego y anuncio evangelizador en clave narrativa y dialogante? La respuesta radica en la creación y recreación vibrante de las estructuras explicativas con las que se comunica la fe en el Dios de Jesucristo. Nuestros jóvenes están saturados de una cultura eclesialmente adulta que habla en términos hieráticos, distintos y distantes a su cultura emergente. Es por ello que:

hemos de facilitar la reelaboración y recreación de nuestro anuncio con nuevos significados, símbolos, paradojas, metáforas, parábolas... Es el anuncio evangelizador tendremos que recobrar un necesario aire de 
gratuidad y alegría, la gratuidad es la actitud vital opuesta al utilitarismo pragmático de la habitual actividad humana. Es la capacidad de contemplación admirativa de saber perder el tiempo, aceptando la vida como don y gracia, en un clima estético y lúdico (homo ludens), como una actitud cultural y humana que abarca todas las actividades personales, sociales y por ende las religiosas. (Liberti, 2007: p. 46)

\section{Finalmente y a modo de conclusión: ¿cómo hablar de Dios a las juventudes?}

En una entrevista que Georg Sporschill S. J. realizó al Cardenal Carlo María Martini S. J. (2008) y que está en el libro "Coloquios nocturnos en Jerusalén" se le preguntaba a Martini cuáles eran las claves que le habían ayudado para hablar de Dios a las juventudes cuando había sido Obispo de Milán. El prelado respondió:

lo que intentamos en la catedral de Milán fue simplemente prestar oídos a textos de la Sagrada Escritura. [...] Yo no daba respuestas previamente preparadas, sino sólo el impulso a escuchar la Palabra, a estar alertas y atentos. Tampoco daba muchos conocimientos exegéticos, sino que me limitaba a intentar que los jóvenes se confrontaran de manera directa con el texto. $\mathrm{Y}$ de ese modo alcanzaron familiaridad con Jesús. Entendieron que Dios los estaba interpelando. [...] Creo que también fue importante la vivencia de comunidad (p. 83)

La experiencia de Martini nos puede dar luces sobre cómo encauzar nuestro trabajo evangelizador por medio del uso de un nuevo lenguaje religioso, pero hay que ser conscientes de que no existen recetas mágicas para poder atraer más jóvenes a la Iglesia, porque caeríamos en el proselitismo. La experiencia de la fe debe ser una respuesta libre a una interpelación que Dios realiza al joven, respuesta que sí debe ser ayudada por la comunidad. Pero ¿qué debe hacer esa comunidad con las juventudes? La respuesta es clara: vivir en constante apertura, o ser una Iglesia "de salida" como nos ha propuesto el Papa Francisco.

¿Cómo evangelizar? ¿Qué lenguaje religioso hemos de utilizar para hacer más creíble un mensaje que en esencia ya es creíble pero que para las juventudes responde muchas veces a una herramienta ideológica ya pasada de moda? ¿Qué les dice a las juventudes el Dios de Jesús de Nazaret? El teólogo Juan Luis 
Segundo (1967) nos recuerda que "las palabras que se usen para evangelizar deben estar cargadas de sentido emocional en el pueblo. El Evangelizador que las usa parte de esta manera de una experiencia humana que le da pie a una profundización mostrando en la esperanza el Mensaje de la Buena Noticia" (10). Se hace necesario por tanto el recurso de la recuperación de los relatos y de las palabras pero también de los silencios forzados, de los jóvenes.

Se debe también considerar el tema de la imaginación como posibilidad de pensar creativamente una Iglesia que asuma los desafíos indignados de los jóvenes por medio de la consideración del lenguaje simbólico, de la narración como configuradora de sentido, desde el diálogo como elemento siempre nuevo de construcción de intersubjetividad y desde el juego y el encuentro como experiencias humanas y eclesiales que favorecen la gratuidad. Hay una riqueza en los jóvenes que debemos aprovechar y permitirles que se conviertan en actores sociales que, desde la fe en Jesucristo, puedan construir un mundo nuevo, justo y bello. Sabemos que los jóvenes se movilizan por temas de solidaridad, de demandas de una educación igualitaria, de mayor representatividad, de respeto por las minorías culturales, sexuales y sociales. Pero es deber del "evangelizador con espíritu" (Papa Francisco) que esta conciencia social se comprenda como la acción de Dios en los signos de los tiempos. Y es allí en donde radica la tarea del lenguaje teológico o religioso, ya que "el lenguaje también tiene la función de ayudar al nacimiento de aquello que todavía está informe y oscuro en el sujeto, para configurarlo y sacarlo a la luz, de tal manera que éste asuma su propia interioridad". (Lenaers, 2008: p. 13)

Al final de este desarrollo, que no pretende ser un recetario del buen evangelizador juvenil, nos quedan más desafíos que respuestas aseguradas. Pero creemos que existe un principio de fe que ilumina el proyecto de un nuevo lenguaje teológico que permita invitar a los jóvenes a la fe. La Encarnación del Verbo, de Jesucristo, es aquello que debe ser el motor de trabajo educativo, pastoral y teológico que nos mueva a desgastar nuestra vida en el trabajo juvenil. La fidelidad al Dios que en Jesucristo habla de una manera nueva y radical pero a la vez comprensible, pasa por el reconocimiento de ese mismo Dios que habita en cada uno de los jóvenes. Solo en el discernimiento hecho desde la fe podremos acoger a Jesucristo al comprender que Él da a conocer a un Dios que se ha hecho próximo a nosotros por medio de su Palabra hecha eterno joven.

\section{Universidad Santo Tomás, Facultad de Teología}




\section{Referencias}

Amozurrutia, J. (2006). Modelos de reflexión sobre el método teológico. Efemérides Mexicana 24, pp. 37-76.

Arteaga, A. (1996). Cada uno escuchaba hablar en su propio lenguaje las maravillas de Dios. Anales de la Sociedad Chilena de Teología, VII, pp. 249-269.

Barriga, C. (1998). Nuestro humilde Dios de cada día. Ensayos de Teología Narrativa. Chile: San Pablo.

Boff, L. (1980). Los sacramentos de la vida. Santander: Sal Terrae.

Duarte, K. (2000). ¿Juventud o juventudes? Acerca de como mirar y remirar a las juventudes de nuestro continente. Última década, 13, pp. 59-77.

Duch, L. (1979). La experiencia religiosa en el contexto de la cultura contemporánea. Madrid: Bruño-Edebé.

Figueroa, M. y Villena, M. (2012). La religiosidad de los jóvenes en tiempos de cambio. En Saavedra, E. (Ed.). Jóvenes, cultura y religion Segundo Informe 2010-2011, pp.17-29.

Irarrázaval, D. (2000). Símbolos y conceptos de Dios. En: Anales Sociedad Chilena de Teología, X, pp. 177-195.

Kasper, W.(2003). El Evangelio de Jesucristo. Santander: Sal Terrae.

Lenaers, R. (2008). Otro cristianismo es posible, fe en lenguaje de modernidad. Ecuador: Abya-Yala.

Liberti, L. (2007). Hacia un anuncio narrativo y dialogal. Teología, 92, pp. 31-49.

Mardones, J. (2003). La vida del símbolo, la dimensión simbólica de la religión. Santander: Sal Terrae

Maccio, C. (1983). El complejo de Edipo, instrumento de liberación. Santander: Sal Terrae.

Martini, C. y Sporschill, G. (s. f.). Coloquios nocturnos en Jerusalén. San Pablo: Navarra.

Morales, J. (2004). Introducción a la teología. Navarra: EUNSA.

Nicolau, M. (1969). Teología del signo sacramental. Madrid: BAC

Ricoeur, P. (1994). Fe y filosofía. Problemas del lenguaje religiosa. Buenos Aires: Almagesto - Docencia.

Segundo, J. L. (1967). Notas sobre lenguaje y evangelización. Revista Pastoral Popular 102, pp. 5-11.

Silva, J. (2006). Jóvenes y religión. Cuando la crisis es motivo de esperanza. Anales de la Sociedad Chilena de Teología, VI, pp. 61-93.

Villa, M. (2011). Del concepto de juventud al de juventudes y al de lo juvenil. Revista Educación y Pedagogía, 23, 147-157. 